Symposium

\title{
Prothesentråger profitieren von Mini-Implantaten
}

Im Kontext des demografischen Wandels steigt die Anzahl an Total- bzw. Teilprothesenträgern in der europäischen Bevölkerung stetig und viele von ihnen klagen über unzureichenden Halt ihres herausnehmbaren Zahnersatzes. Da der mangelnde Prothesenhalt mit deutlichen Einschränkungen der Lebensqualität verbunden ist, ist es von zentraler Bedeutung, entsprechende, auf die Bedürfnisse dieser Patienten abgestimmte Therapiekonzepte zu entwickeln. Inwiefern MiniImplantate eine geeignete Therapieoption darstellen, wurde anlässlich eines Symposiums am 23. November 2013 in München erörtert. Dabei herrschte Einigkeit darüber, dass sowohl die klinischen Erfahrungen als auch die mittlerweile vorhandenen Studien für den Einsatz von Mini-Implantaten im teil- und unbezahnten Kiefer sprechen. $\mathrm{Zu}$ den Referenten des von $3 \mathrm{M}$ ESPE organisierten Symposiums mit dem Titel „Mini-Implantate im Zeichen des demografischen Wandels“ gehörten sowohl Vertreter unterschied-

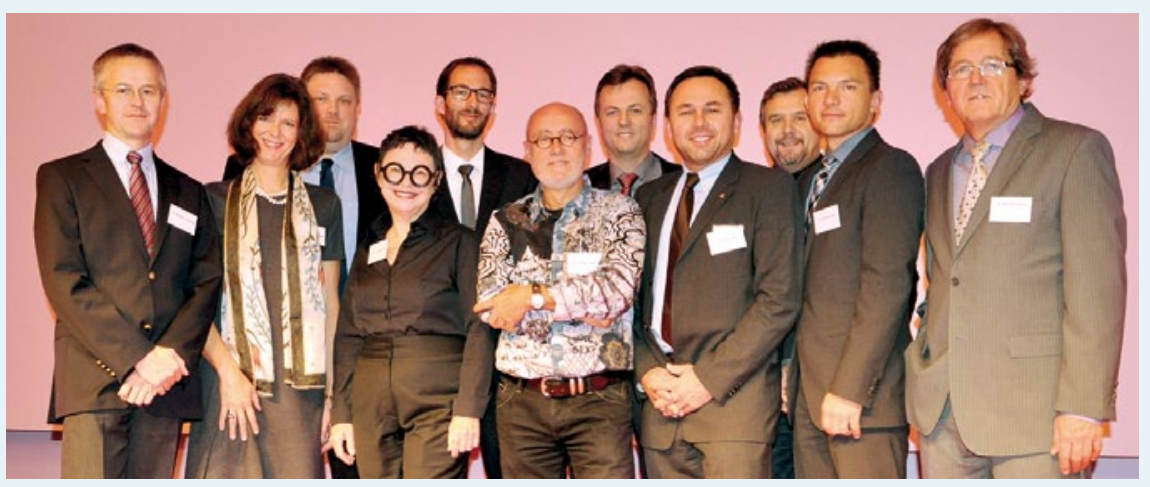

Die Referenten des MDI-Symposiums (v.l.n.r.): Dr. W. Tamminga, die Moderatoren Prof. Dr. F. Müller und Dr. K. Schwarz, Prof. Dr. J. Feine, Dr. A. Worni, Dr. J. Hilgert, PD Dr. F. Heinemann, Dr. J. Z. Gal, T. Hauck, Dr. Z. Keilinger und Dr. W. Walzer.

licher Universitäten als auch niedergelassene Zahnärzte. Die Teilnehmer waren begeistert von der Qualität der Vorträge, der Themenvielfalt und der Möglichkeit, nicht nur im Rahmen einer Posterpräsentation mit den Referenten persönlich in Kontakt zu treten.

Nach einer Pressemitteilung der 3 M Deutschland GmbH, Seefeld

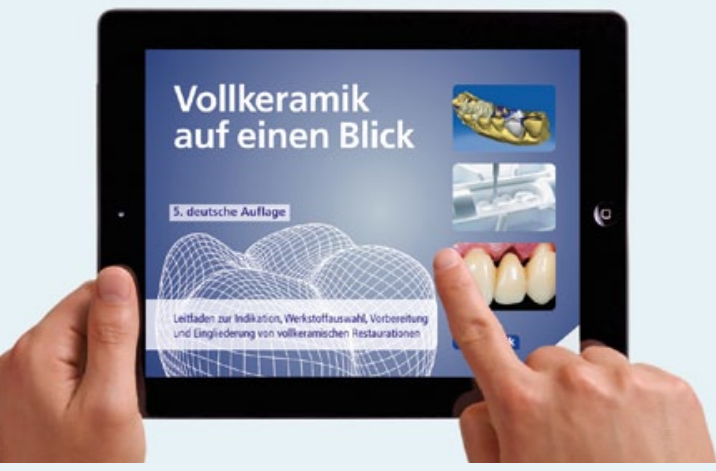

In 2006 erstmalig aufgelegt, ist das Buch „Vollkeramik auf einen Blick“ - ein zahnärztlicher Leitfaden zur Indikation, Werkstoffauswahl und Eingliederung vollkeramischer Restaurationen - mehrfach erweitert und nachgedruckt worden. Nun steht das Keramikhandbuch auch als eBook über die AG Keramik-APP zum Download für das iPad in der Apple-Version zur Verfügung. Die Androiden-Version wird in Kürze bereitgestellt werden. Damit können sich die Nutzer der APP an
Universitäten, in Fortbildungseinrichtungen, in der Praxis, als Doktorand, Referent, Zahntechniker oder Fachjournalist in Sekunden über den aktuellen Stand der Restauration mit Vollkeramikwerkstoffen interaktiv informieren. Das Buch wurde von erfahrenen Autoren wie den Professoren Frankenberger, Kern, Kohal, Kunzelmann, Mehl, Pospiech sowie Dres. Reiss und Wiedhahn verfasst und hat damit das gesamte aktuelle Fachwissen zur vollkeramischen Restauration gebündelt. Das eBook enthält 152 Seiten, ist reichhaltig illustriert und thematisch klar und übersichtlich gegliedert. Über die Website www.ag-keramik.de/aktuelles wird der Interessent mit dem iTunes APP-Store verbunden. Dort ist die AG Keramik-APP als kostenloses Download zu finden. Das komplette eBook kann dann für 14,99€ heruntergeladen werden.

Nach einer Pressemitteilung der AG Keramik, Ettlingen

\section{Diagnostik}

Diagnostik-Technik \section{gewinnt}

\section{Innovationspreis}

Knapp 4000 teilnehmende Zahnärztinnen und Zahnärzte in Deutschland wählten die KaVo DIAGNOcam zum innovativsten Dentalprodukt des Jahres 2013. Damit konnte sich das Unternehmen erfolgreich gegen 6 Wettbewerbsprodukte durchsetzen.

Das neue Karies-Diagnoseverfahren ist das 1. Kamerasystem, das die Strukturen des Zahnes nutzt, um die Kariesdiagnose abzusichern. Dazu wird der Zahn mit einem Licht durchschienen (transilluminiert) und wie ein Lichtleiter benutzt. Eine digitale Videokamera erfasst das Bild und macht es live auf einem Computerbildschirm sichtbar. Kariöse Läsionen werden dann als dunkle Schattierungen dargestellt. Die erfassten Bilder können abgespeichert werden und vereinfachen dadurch deutlich das Monitoring bzw. die Patientenkommunikation.

Das Kamerasystem bietet mit seiner DIFOTI-Technologie (Digital Imaging Fiberoptic Transillumination) eine hohe diagnostische Sicherheit, die der Röntgendiagnose in vielen Fällen vergleichbar bzw. überlegen ist, insbesondere bei Approximal- und Okklusalkaries. Das röntgenstrahlungsfreie Gerät ermöglicht somit eine frühe und sehr schonende Karieserkennung.

Nach einer Pressmitteilung der KaVo Dental GmbH, Biberach / Riss 


\section{Neue Produkt-}

\section{und Preisstrategie}

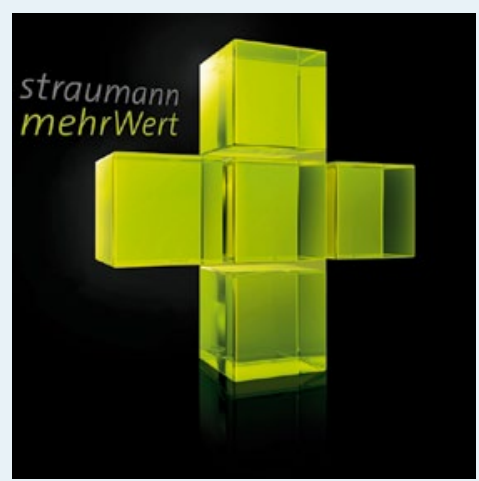

Straumann startet die Initiative mehrWert+. Kunden und Patienten sollen von einem verbesserten Preis/Leistungs-Verhältnis bei Zahnimplantaten profitieren. Das Hochleistungsmaterial Roxolid ersetzt ab sofort Titan als Standardmaterial bei Straumann-Implantaten - ohne Mehrkosten. Für Kunden und Patienten bedeutet dies: sie erhalten mit Roxolid das beste Implantatmaterial in Kombination mit der SLActive- oder SLA-Oberfläche zum gleichen oder tieferen Preis wie bislang das entsprechende Titan-Implantat. Für preissensitive Patienten bietet Straumann weiterhin ein im Umfang reduziertes Titan SLA-Sortiment zu attraktiven Konditionen an.

Wolfgang Becker, Geschäftsführer Straumann, ist überzeugt: „Mit mehrWert+ setzen wir auf unser bestes Implantatmaterial. Roxolid ist einzigartig im Hinblick auf Festigkeit und Osseointegrationseigenschaften. Behandler und Patienten profitieren gleichermaßen von dem kostenlosen Material-Upgrade von Titan auf Roxolid. Bei uns wählt der Behandler zwischen 2 Oberflächen, 2 Materialien und 3 Preisen.“

Nach einer Pressmitteilung der Straumann GmbH, Freiburg Internet: http://www.straumann.de/ mehrwert-plus
Kieferorthopädie

\section{Selbstligierende Bracket-Systeme Mini Metal Brackets}

Mit Gründung der GC Orthodontics Europe $\mathrm{GmbH}$ beschreitet die GC Corporation einen weiteren Schritt auf dem Weg hin zum Ziel, ein umfassendes Service- und Beratungspaket für den kieferorthopädischen Sektor anzubieten. Die Bracket-Systeme der Experience Linie reihen sich in dieser Hinsicht in die Produktpalette ein. Je nach Behandlungsmethode besteht die Möglichkeit, zwischen aktiven und passiven Brackets zu wählen, welche mit einer mikrosandgestrahlten Klebebasis sowie einer disto-gingivalen Farbcodierung ausgestattet sind - was die Arbeit für den Kieferorthopäden erheblich erleichtert. Die genannten Vorteile vereinen alle Brackets der Experience Linie - zu welcher neben Experience Metall, Experience Ceramic und Experience Lingual auch die Experience Mini Metal-Brackets zählen. Letztere stellen in der ästhetischen, Rhodium-beschichteten Variante Experience Mini Metal Rhodium eine Premiere in der festsitzenden Kieferorthopädie dar: Kaum höher als ein konventionelles Bracket, verei-

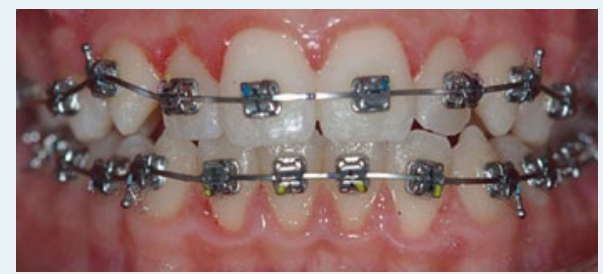

nen sie Unauffälligkeit und Komfort auf effektive Weise. Ein grundlegender Aspekt ist die Beständigkeit des Metalls, welche in Verbindung mit der behandelten Oberfläche den anspruchsvollen Anforderungen an Material und Leistung gerecht werden kann. Die Mini Metal Rhodium-Brackets eröffnen dem Kieferorthopäden somit eine Therapiemöglichkeit, die gleichermaßen den Anforderungen moderner Behandlungsmethoden wie auch den ästhetischen Ansprüchen der Patienten entgegen kommt.

Nach einer Pressemitteilung der GC Orthodontics Europe GmbH, Augsburg Internet: www.gcorthodontics.eu

\section{Implantologie}

\section{Hohlraumversiegelung als Pråventions- maßnahme für Implantate}

Eine Besiedelung der Implantatinnenräume mit Keimen aus der Mundhöhle ist ohne entsprechende Maßnahmen unvermeidbar und erhöht das Risiko einer Ansammlung von Bakterien, Viren und Pilzen, für die die feuchtwarme Umgebung ein perfektes Milieu darstellt. GapSeal ${ }^{\circledR}$ (Hager\&Werken) versiegelt prophylaktisch diese Spalten und füllt entstehende Hohlräume dauerhaft aus. Es verhindert wirksam das Eindringen von Keimen in die Hohlräume und vermindert die Reinfektion des periimplantären Gewebes, das die Hauptursache für die Entwicklung periimplantärer Erkrankungen darstellt. Das Material besteht aus einem speziell abgestimmten Silikonmatrix-Wirkstoffkomplex, der durch das Abdichten der Hohlräume ein Eindringen von Bakterien verhindert. Das hochvisköse Silikon wird direkt nach der Insertion des Implantates beim ersten Eindrehen der Verschlussschraube angewendet. Durch sein hydrophobes Verhalten und seine Standfestigkeit wird es nicht ausgewaschen und verbleibt so dauerhaft in situ. Die Anwendung erfolgt durch kleine Portionstips steril und komfortabel. Diese applizieren das Versiegelungsmaterial mit einem speziellen, autoklavierbaren Spender schnell und hygienisch. Das Produkt kann inzwischen auf eine klinische Erfahrung von 16 Jahren zurückblicken.

Nach einer Pressemitteilung der Hager \& Werken GmbH \& Co. KG, Duisburg

Internet: www.hagerwerken.de 
Fort- und Weiterbildung

\section{Sicher in die}

digitale Zukunft

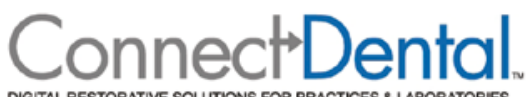

Digitale Zahnheilkunde bedeutet neue Möglichkeiten und Chancen in der $\mathrm{Zu}$ sammenarbeit zwischen Zahnarztpraxis und Dentallabor, verbunden mit einer Steigerung des Komforts für die Patienten und der Effektivität im gesamten Behandlungsablauf. Doch das Produktangebot in der digitalen Zahnmedizin ist groß und eine allgemeingültige Lösung für den Zahnarzt oder das Labor gibt es nicht. Hier besteht Bedarf an einer Systemlösung, die den gesamten Prozess im Auge hat und dessen Funktionalität sicherstellen kann. Dieser Ansatz ist einer der Hauptschwerpunkte von Henry Schein bei dem unternehmenseigenen Komplettlösungsangebot ConnectDental. Mithilfe eines bundesweiten Spezialisten-Teams finden hierzu Beratungen vor Ort und innerhalb von Fortbildungsveranstaltungen statt. Um Zahnärzten und Laborinhabern die Möglichkeit zu geben, auf dem Markt gängige Geräte und die damit verbundenen Arbeitsvorgänge eigenständig, live und unter fachkundiger Beratung durch die Henry Schein-Spezialisten und die CAD / CAM-Hersteller auszuprobieren, fand im November im Hamburger Dentalen Informations Center (DIC) von Henry Schein der 1. CAD / CAMSystemvergleichs-Workshop statt.

In Zirkeltrainings konnten sich die Teilnehmer aus Praxis und Labor einen Eindruck über die Vielzahl der vorhandenen Systeme und ihrer Vorzüge verschaffen. Zum Abschluss hatten die Teilnehmer die Möglichkeit, das von ihnen bevorzugte System selbst zu testen. Aufgrund der hohen Nachfrage und des großen Erfolgs werden am 22. März und am 20. September 2014 weitere Workshops in Hamburg stattfinden. Diese Workshops und zusätzliche Fortbildungsveranstaltungen zum Thema digitale Zahnheilkunde werden von Henry Schein bundesweit auch in anderen DICs des Unternehmens angeboten.

Nach einer Pressemitteilung der

Henry Schein Dental Deutschland GmbH, Langen

Internet: www.henryschein-dental.de,

www.henryschein.com
Implantologie

\section{Optimierte Implantatnachsorge mit flexiblen Kunststoffsonden}

Über die Nachsorge von Implantaten wurde in der Vergangenheit viel kontrovers diskutiert. Die früher vehement vertretene „No-Touch-Policy“ scheint in Zeiten steigender Anzahl periimplantärer Erkrankungen passé. Inzwischen ist man sich einig: In einem 1. Schritt wird zunächst sanft der Sulkus ausgestrichen um bei eventuellen ersten Anzeichen einer Entzündung oder Blutung auch zu Sondieren und eine mögliche Taschentiefe zu messen. Um das relativ weiche Titan der Implantate jedoch nicht weiter anzurauen empfiehlt es sich, ausschließlich mit flexiblen Sonden aus Kunststoff zu arbeiten. Diese bieten den Vorteil der Flexibilität, sodass sie sich auch an bauchigen Implantaten oder Prothetiküberhängen gefahrfrei und substanzschonend einsetzen lassen. Das weiche Material vermeidet zudem das Risiko weitere plaqueakkumulative Furchen zu setzen.

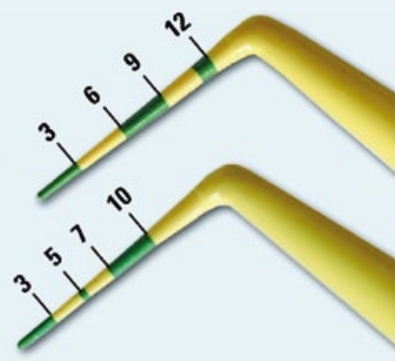

Es ist zu empfehlen für jedes Implantat eine eigene Sonde $\mathrm{zu}$ verwenden. Andernfalls besteht das Risiko der bakteriellen Übertragung aus einem gefährdeten Saum in einen bis dato noch völlig unvorbelasteten Bereich. Die flexiblen PDT-Kunststoff-Sonden sind exklusiv über die Zantomed $\mathrm{GmbH}$ erhältlich in verschiedenen Skalierungen (Art. T212: 3,6,9,12 mm; Art. T214: 3,5,7,10 mm; Art. T216: 1-12 je $1 \mathrm{~mm}$ ).

Nach einer Pressemitteilung der Zantomed GmbH, Duisburg Internet: www.zantomed.de

\section{Endodontie \\ Studie* zeigt: Apexlokator genauer als DVT}

Die elektrische Längenbestimmung hat sich in den letzten Jahren als Goldstandard etabliert. Auch die DGZMK bestätigt in einer Stellungnahme die Überlegenheit dieser Methode gegenüber der röntgenologischen Bestimmung. Mit 3D-Röntgen (DVT: Digitale Volumentomografie) steht inzwischen eine weitere Technologie zur Verfügung, mit der sich die endodontische Arbeitslänge bestimmen lässt. Eine an der Universität Granada/Spanien durchgeführte Studie befasst sich mit der Frage, welche dieser modernen Methoden die zuverlässigeren Ergebnisse liefert. Dazu wurden 150 extrahierte Zähne in 5 Gruppen eingeteilt. 4 Gruppen dienten der Auswertung von Messergebnissen der elektrischen Längenbestimmung mit dem Apexlokator Raypex 6 im trockenen Kanal (1) bzw. mit 3 verschiedenen Flüssigkeiten (2-4). Es wurden jeweils das Foramen Major und die apikale Konstriktion als Messpunkte definiert. In Kontrollgruppe 5

\footnotetext{
* Original Studie: http://onlinelibrary.wiley.com/ doi/10.1111/iej.12140/abstract
}

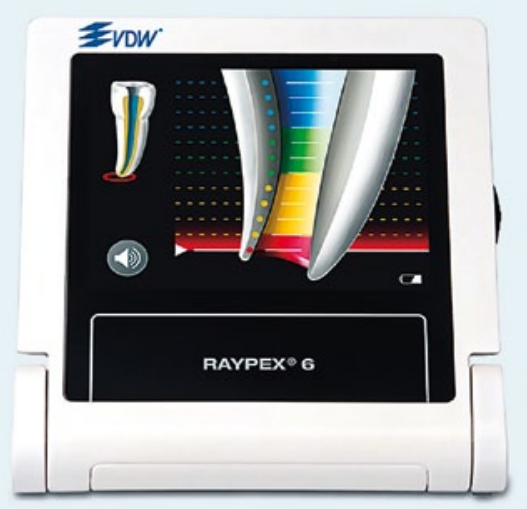

wurden die Ergebnisse der Längenbestimmung mit DVT ausgewertet.

Im Resultat waren die elektronischen Messergebnisse zuverlässiger als die Bestimmung der Arbeitslänge durch DVTScans, insbesondere bei der Bestimmung des Foramen Major. Die Studie bestätigt, dass Raypex 6 bei der Bestimmung der Arbeitslänge präziser und verlässlicher als die digitale Volumentomografie ist.

Nach einer Pressemitteilung der VDW GmbH, München

Internet: www.vdw-dental.com 


\section{Adhäsiv - 3 Åtztechniken}

Das 1-Flaschen-Adhäsiv xeno Select bietet dem Zahnarzt jetzt die freie Wahl der Ätztechnik. Je nach Indikation kann es sowohl selbstätzend, mit selektiver Schmelzätzung oder in der Etch\&Rinse-Technik eingesetzt werden. Dadurch erübrigt sich eine Vorratshaltung unterschiedlicher Adhäsive. Dabei zeigt das Produkt bei jeder Technik eine hohe Leistung und sorgt für einen

\footnotetext{
Latta MA; 2013 (auf Anfrage bei Dentsply erhältlich)
}

starken und zuverlässigen Haftverbund auf Schmelz und Dentin. Doch Flexibilität und Wahlfreiheit sind nicht alles. Neben der Vereinfachung zeigt das Produkt aufgrund seiner besonderen Komposition hohe und besonders ausgewogene Haftwerte auf Schmelz und Dentin. Die Haftwerte ermittelte unter anderem der renommierte Prof. Dr. Mark Latta, Dekan und Professor der Zahnmedizin an der Creighton Universität in Omaha, Nebraska. Er konnte hohe und gleichzeitig ausgewogene Haftwerte so-

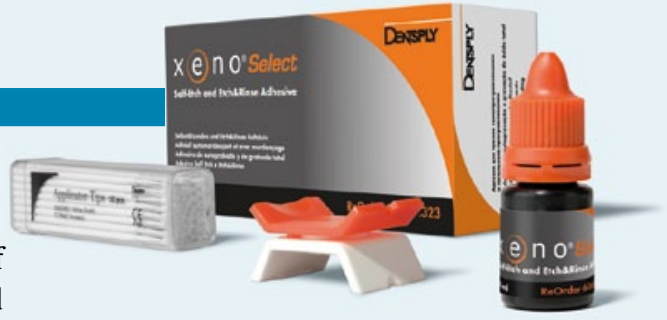

wohl für die Etch\&Rinse-Technik als auch für die Verwendung von xeno Select als selbstätzendes Adhäsiv nachweisen ${ }^{1}$.

Dank des einzigartigen Lösungsmittelsystems ist xeno Select zudem ein zuverlässiger und robuster Partner im zahnärztlichen Alltag.

Nach einer Pressemitteilung der Dentsply DeTrey GmbH, Konstanz Internet: www.dentsply.de

\section{Praxistest}

\section{Fluoridhaltiger Schutzlack überzeugt Zahnärzte}

Von Oktober 2012 bis Juli 2013 haben Zahnärzte in verschiedenen Ländern den neuen fluoridhaltigen Schutzlack Fluor Protector S von Ivoclar Vivadent getestet. Insgesamt wurden 251 Fragebögen ausgewertet. Dabei hat es sich gezeigt, dass der fluoridhaltige Schutzlack regelmässig zur Fluoridierung mehrerer Zähne (74\%), eines kompletten Gebisses (68\%) oder eines Einzelzahnes (63\%) eingesetzt wird. Die Eigenschaften der Dosiertube hat mehr als $90 \%$ der Zahnärzte überzeugt: kein Verkleben des Verschlusses, kein Nachlaufen, hygienische Darreichung, bequemes Ausdrücken und präzises Dosieren.

$85 \%$ der Zahnärzte waren mit den Eigenschaften und der einfachen Handhabung des Produktes zufrieden. 79\% der Zahnärzte waren zufrieden mit der Akzeptanz bei Patienten. 88\% der Zahnärzte werden den Schutzlack künftig in ihrer Praxis verwenden. Die Studie hat somit die Vorteile des Lacks bestätigt: Er ist als homogene Lösung direkt verfügbar und lässt sich mit der Dosiertube gut abmessen. Zudem ermöglicht er eine gezielte Applikation und hat eine gute Haftung.

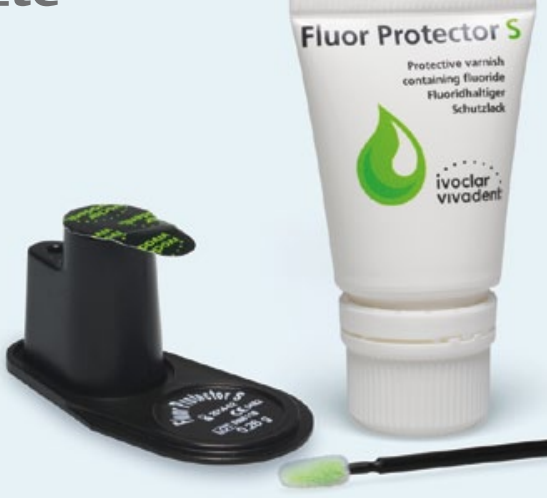

Nach einer Pressemitteilung der Ivoclar Vivadent AG, FL-Schaan Internet: www.ivoclarvivadent.com

\section{Präzise Bilder verbessern Diagnostik und Workflow}

Je besser die Details auf einem zahnärztlichen Röntgenbild sichtbar werden, desto exakter und schneller kann der Behandler die Therapie planen. Deshalb hat Dürr Dental in die Konzeption eines neuen Panoramaröntgengeräts investiert. In Bezug auf die Bildschärfe setzt das Unternehmen beim VistaPano auf die S-Pan-Technologie. Im Vergleich zu anderen neueren Panoramageräten, die eine manuelle Auswahl der schärfsten Bereiche erfordern, geht die neue Technologie noch einen Schritt weiter: aus mehreren, gleichzeitig erfassten Schichtlagen werden jeweils in horizontaler sowie vertikaler Richtung die aussagekräftigsten Schichtlagen automatisch identifiziert und zu einer Aufnahme verschmolzen. Dies führt dazu, dass alle wichtigen diagnostischen Informationen wie beispielsweise Wurzeln im Unter- als auch Oberkiefer scharf dargestellt werden, der Mandibularkanal gut sichtbar ist und auch die Knochenstruktur Aussagekraft besitzt.

Das Gerät verfügt ferner über einen Schnellscan-Modus, der es dem Praxispersonal ermöglicht, eine Panoramaaufnahme innerhalb von nur 7s anzufertigen. Das heißt, der Patient wird der geringstmöglichen Strahlendosis ausgesetzt. Die gute Bildqualität trotz geringer Strahlendosis wird durch einen modernen CsISensor ermöglicht. Dies gilt gleichermaßen für den Kinderaufnahmemodus mit verkleinertem Belichtungsbereich, der die Dosis um bis zu $56 \%$ reduziert.

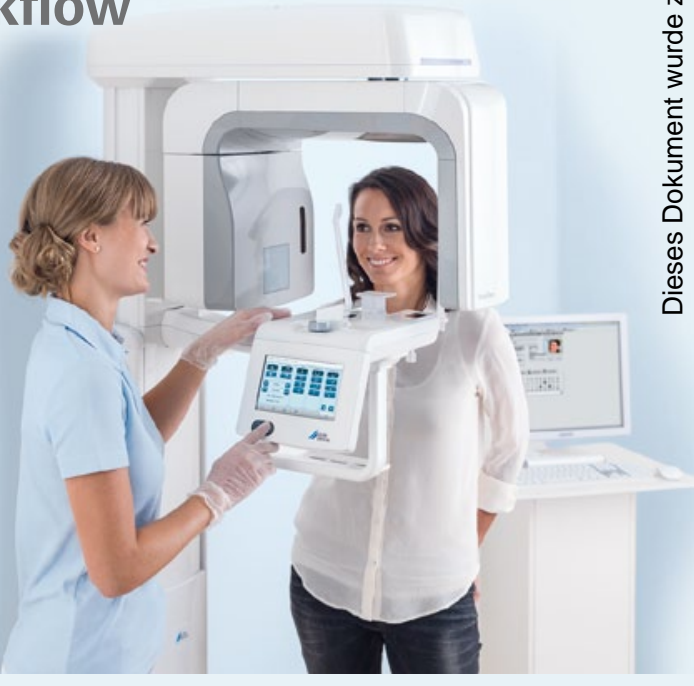

Nach einer Pressemitteilung der DÜRR DENTAL AG, Bietigheim-Bissingen Internet: www.duerr.de 
Workshop

Neue digitale

Verfahren in der

Implantatprothetik

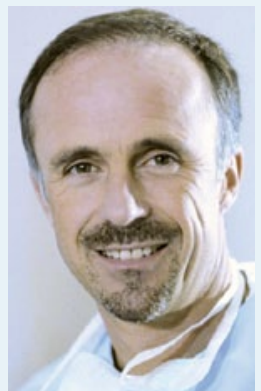

A. Valentin

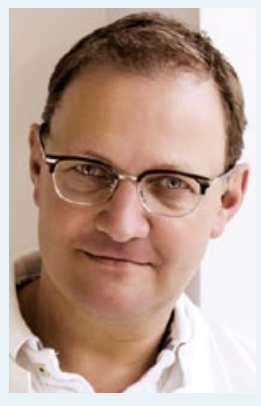

M. Engelschalk
BIOMET3i hat sich auf dem diesjährigen Kongress der DGI nicht nur mit seinem umfassenden Produktsortiment am Ausstellungsstand der Implant Expo präsentiert, sondern auch mit einem Workshop zum Thema „Die digitale Abformung - ein neuer Workflow für höhere Präzision und Erfolg in der Implantatprothetik“.

Digitale Technologien ermöglichen neue Behandlungsprozesse in der Zahnheilkunde, die insbesondere in der Implantologie und Implantatprothetik zu einer höheren Präzision, einer Verbesserung der Ästhetik und einer Steigerung der klinischen Erfolgsrate beitragen können. Diesen Themenkomplex beleuchteten die beiden Referenten Prof. Dr. Dr. Andreas Valentin, Mannheim (Thema: Eleganz in der Implantat-Ästhetik - das neue Encode-Verfahren) und Dr. Marcus Engelschalk, München (Digitaler intraoraler Scan von Implantaten auf Basis des Gingivaformers - ein neuer Workflow für höhere Präzision und Erfolg in der Implantatprothetik) in ihrem jeweiligen Vortrag anhand von zahlreichen Beispielen aus ihrer Praxis. An der lebhaften Diskussion und dem fachlichen Austausch nach den Vorträgen wurde deutlich, wie wertvoll die klinischen Erfahrungen und detaillierten Falldarstellungen der beiden Referenten für die Teilnehmer waren.

Nach einer Pressemitteilung der BIOMET 3i Deutschland GmbH, München

\section{Prothetik \\ Über 10 Millionen Mal im Einsatz}

5 Jahre nach seiner Einführung übertrifft das provisorische Kronen- und Brückenmaterial Protemp 4 von $3 \mathrm{M}$ ESPE die Erwartungen. Nun konnte bekannt gegeben werden, dass weltweit bereits mehr als 10 Millionen temporäre Versorgungen mit dem sowohl für den Kurz- als auch Langzeiteinsatz geeigneten Bis-AcrylKomposit hergestellt wurden. Dies bestätigt einmal mehr, dass das Material Anwendern die Zuverlässigkeit und Qualität liefert, die sie für die provisorische Versorgung als äußerst wichtigen Schritt im Rahmen restaurativer Behandlungen benötigen.

Dank der speziellen Zusammensetzung bietet das Bis-Acryl-Komposit eine hohe Biegefestigkeit. Diese und andere klinische Eigenschaften wie z.B. die einfache Anwendung, hohe Widerstands-

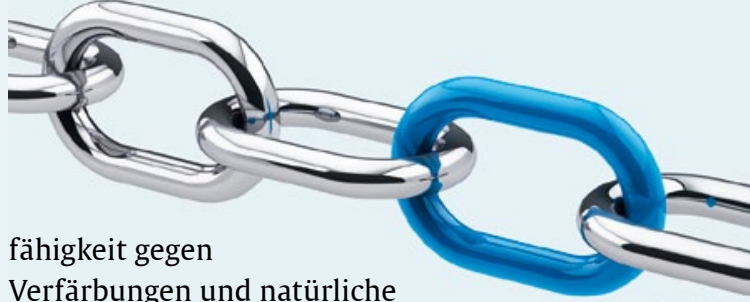

Verfärbungen und natürliche

Fluoreszenz wurden bereits in zahlreichen Veröffentlichungen in unabhängigen Publikationen bestätigt. $\mathrm{Zu}$ den besonderen Vorteilen gehört außerdem, dass bei diesem Komposit keine Politur oder Glasur erfolgen muss, wodurch Zeit eingespart wird: Die Oberfläche wird einfach mit Alkohol gereinigt, um einen natürlich wirkenden, langanhaltenden Glanz zu erzielen, der auch zu einer einfachen Plaqueentfernung beiträgt.

Nach einer Pressemitteilung der

$3 \mathrm{M}$ Deutschland AG, Seefeld

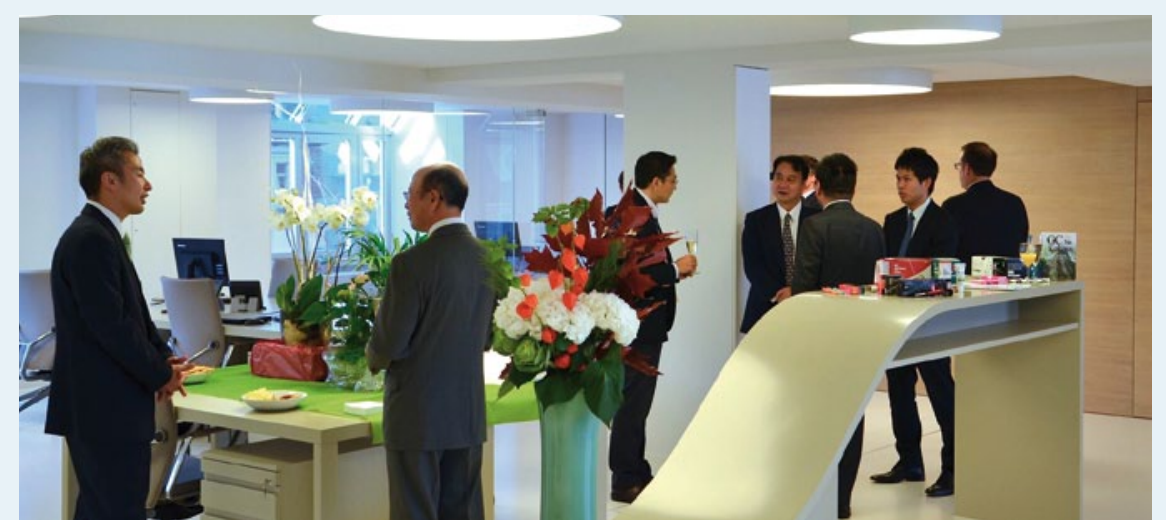

Zur Koordination der internationalen Aufgaben hat GC 2013 ein Büro in Luzern eröffnet. Von hier aus sollen die Kunden in den Regionen Europa, Asien und Amerika noch effektiver an die neuen Produktentwicklungen herangeführt werden. Am Sitz der neuen Niederlassung in der Schweizer Kulturstadt Luzern begrüßte Makoto Nakao, President und CEO der GC International AG sowie Chairman und CEO der GC Corporation, die geladenen Gäste aus Wissenschaft, Politik und Handel. Hierfür bot der Festsaal des Hotels Astoria am 9. September 2013 den Rahmen. Makoto Nakao skizzierte das Leitmotiv des multinationalen Unternehmens, im- mer bessere Produkte zu entwickeln und somit dem Patienten eine immer höhere Lebensqualität zu bieten. Aufgabe des Unternehmens ist es, die Entwicklung neuer Produkte global mit Fokus auf die regionalen Märkte zu koordinieren. In Luzern erfolgt die Abstimmung mit GC Europe, GC America und GC Asia. Unterstützt wird das Engagement von Makoto Nakao von weltweit rund 2300 Mitarbeitern. Sitz der GC Europe ist Leuven/Belgien, während die GC Germany GmbH in Bad Homburg beheimatet ist.

Nach einer Pressemitteilung der GC Europe N.V., B - Leuven Internet: www.gceurope.com 